\title{
Preparing the sexual health workforce to deliver integrated services: is education the answer? A qualitative study exploring the impact of sexual health education on developing integrated policy and practice
}

\author{
Judy Brook ${ }^{1}$, Debra Salmon ${ }^{2}$ and Rachael-Anne Knight ${ }^{3}$ \\ ${ }^{1}$ Lecturer in Health Visiting, School of Health Sciences, Division of Health Services Research and Management, City, \\ University of London, Northampton Square, London, UK \\ ${ }^{2}$ Deputy Dean, School of Health Sciences, City, University of London, Northampton Square, London, UK \\ ${ }^{3}$ Associate Dean for Education Technology and Innovation, School of Health Sciences, City, University of London, \\ Northampton Square, London, UK
}

\begin{abstract}
Aim: This study aimed to explore the ability of sexual health nurses working in the South West of England, to implement new learning within existing sexual health service delivery models. Drawing on Lipsky's account of street-level bureaucracy to conceptualise policy implementation, the impact of workforce learning on the development of integrated services across this region of the United Kingdom was assessed. Background: In order to achieve the United Nations' goal of universal access to sexual health, it is essential for reproductive and sexual health, including HIV provision, to integrate into a single service. This integration requires a commitment to collaboration by service commissioners and an alignment of principles and values across sexual health and contraceptive services. UK health policy has embraced this holistic agenda but moves towards integrating historically separate clinical services, has presented significant workforce development challenges and influenced policy success. Methods: Employing a qualitative approach, the study included data from semistructured telephone interviews and focus groups, and longitudinal data from pre- and post-intervention surveys, collected between September 2013 and September 2015. Data were collected from 88 nurses undertaking a workforce development programme and six of their service managers. Data were analysed using thematic analysis to identify consistent themes. Findings: Nurses confirmed the role of new learning in enabling them to negotiate the political landscape but expressed frustration at their lack of agency in the integration agenda, exposing a clear dichotomy between the intentions of policy and the reality of practice. Nevertheless, using high levels of professional judgement and discretion practitioners managed the incongruence between policy and practice in order to deliver integrated services in the interests of patients. Workforce education, while essential for the transition to the delivery of integrated services, was insufficient to fulfil the sexual health agenda without a strengthening of public health.
\end{abstract}

Key words: education; integrated services; leadership; sexual health nurses

Received 21 July 2016; revised 2 December 2016; accepted 7 February 2017;

first published online 14 March 2017

Correspondence to: Judy Brook, Lecturer in Health Visiting, School of Health Sciences, Division of Health Services Research and Management, City University London, Northampton Square, London EC1V 0HB, UK. Email: Judy.brook@city.ac.uk

(C) Cambridge University Press 2017 


\section{Introduction and policy context}

From a global perspective, the degree to which individuals are free to engage with and enjoy their sexual and reproductive health is inextricably linked with issues of inequality, disadvantage and human rights. International sexual health is highly political in nature, immersed in debates around morality, religion and social value, and is heavily influenced by tensions in the global health context. Traditional approaches of disease control and prevention, and decisions to invest in the targeting of single diseases [such as sexually transmitted infections (STIs) or HIV/AIDS], are often siloed, highly specialised and potentially undermine, rather than strengthen, public health systems. This dominance of large, funded, single-disease programmes can lead to a marginalisation of the importance of the social determinants of health in global health policy (Allotey et al., 2011), and there are significant opportunities for national leadership to promote a more holistic approach to improving the sexual health of communities (Ivankovich et al., 2013).

National leadership and political contexts are key to public health strategy; effecting change in populations cannot be conducted independently of the government, policy and power. To develop a public health workforce, practitioners need to be practically competent and also aware of the social determinants of health, the influence of social values and individual rights on health and wellbeing and have a propensity towards holistic understanding of the individual within their community context. Anderson (2014) argues that it is essential for reproductive health, sexual health and HIV provision to be integrated in to one service to meet the needs of populations, but adds that integrated services can only be developed if there is a commitment to meticulous collaboration and an alignment of principles and values. This is a challenge and it is therefore unsurprising that few countries have achieved a significant evolution towards integrated sexual health services (Dickinson et al., 2009). Smit et al. (2012) identified a clear consensus in South Africa between expert informants in the Department of Health (DH), Non-Government Organisations and universities about the need for integrated sexual health services but multiple complex and inter-related factors at both policy and service level posed barriers to realisation of this aspiration. In Uganda, demographic and socioeconomic characteristics still greatly influence the utilisation of integrated sexual health services by women (Rutaremwa and Kabagenyi, 2016). Temmerman et al. (2014) express frustration at the delay between policy development and policy implementation, citing the 20 year lag between the goal of achieving universal access to reproductive health being set by the United Nations and the current position where this remains an unfinished agenda. It is therefore testing to educate a workforce in anticipation of change in service delivery, especially when confidence in the realisation of that change may be low. However, practitioners are policy actors; drawing on Lipsky's (1980) concept of street-level bureaucracy it is possible to analyse how practitioners 'subvert' systems in order to develop their agendas within the broader policy and financial restraints, and failures to invest in widespread integration. This paper reports on a study that examined issues of integration, from the perspective of a group of sexual health nurses and their managers, who reflected on the challenges to providing integrated sexual health services to their patients.

\section{Background}

UK health policy has embraced the holistic and integrated agenda, recognising that sexual illhealth impacts on all parts of society [DH, 2013a, 2013b; Public Health England (PHE), 2015]. Historically, services in the United Kingdom have been delivered either through Family Planning clinics for contraceptive services or Genito-Urinary (GUM) clinics for sexual health issues. Within the Integrated Sexual Health Services, National Health Service (NHS) Specification emphasis is placed on an integrated service, at a 'one stop shop', delivered by a single health professional (DH, 2013b). The argument for such service models is compelling, however, this move has significant implications for the sexual health workforce. Kane and Wellings (2003) and 10 years later Anderson (2014) acknowledge progress made but concede that without whole system commissioning, the divide between the specialisms with regard to culture and structure is still evident. This is reiterated by PHE (2015), advocating that commissioners, 
providers and wider stakeholders need to take collective responsibility for ensuring accessibility to services. The result is a public health specialism that is in a state of flux, with regional and, in some cases, local differences between service delivery models, mirroring the global dichotomy between a focus on single-disease targeting and a holistic approach to sexual and reproductive health.

\section{Setting}

This paper reports on a study that was commissioned by the South West Regional Health Education Authority to evaluate the implementation of a workforce development programme designed to equip sexual health nurses with the appropriate knowledge and skills to deliver an integrated sexual health service. This programme, designed and run successfully in another region of the United Kingdom (McNall and Kain, 2010), was devised as part of a whole system approach to integrated sexual health service delivery, focussing on practice development and person-centred care. The overall ambition was not only to develop nurses who practise to excellent clinical standards but who also practise across all areas of sexual health service provision as highlighted by national health policy (DH, 2013a). The South West is a region that is geographically diverse, consisting of large cities and rural villages that include both some of the most affluent and the most socially disadvantaged communities across the United Kingdom. These differences in social inequality are reflected in significant gradients in terms of teenage pregnancy rates, STIs and late diagnosis of HIV (Public Health England, 2014).

The specific aim was to explore the ability of sexual health nurses to implement their new learning within existing sexual health service delivery models and assess the impact on the implementation or further development of integrated services across the South West region.

The objectives of the research were to explore:

1. The extent to which nurses' perceptions of their confidence to practise, preparedness and selfefficacy improved through participation in the workforce development programme and the subsequent impact on patient care

Primary Health Care Research \& Development 2017; 18: 270-281
2. The extent to which nurses are able to practice according to the policy agenda of integrated sexual health services

3. Stakeholders' views of the extent to which the educational delivery meets service demands in relation to integrated sexual health provision for patients within the current context

\section{Methods}

A qualitative approach was used in order to elicit the experiences and opinions of the participants. Initial data collection using questionnaires informed the secondary data collection via focus groups and interviews.

\section{Ethics}

The authors assert that all procedures contributing to this work comply with the ethical standards of British Educational Research Associations (BERA) Ethical Guidelines for Educational research (2011) and with the Helsinki Declaration of 1975, as revised in 2008. Ethical approval was obtained from the University of the West of England Bristol Research Ethics University and Faculty Committee prior to the research start dates. In accordance with ethical principles full, informed consent was obtained at all stages of the study after the participants had read the information sheet, in which the voluntary nature of participation and the right to withdraw were emphasised. The data collected were anonymised and research numbers allocated to protect the identity of those who took part.

\section{Sample}

The workforce development programme consisted of two educational modules, delivered by a combination of face-to-face teaching at university and e-learning supported by practical competence assessment in practice placements. Purposive sampling was employed to invite all nurses who undertook the workforce development programme $(n=89)$ to take part in the study. In total, 88 nurses were recruited to the study across four cohorts between November 2013 and October 2015. 


\section{Questionnaire}

Complete pre- and post-questionnaire data were collected from 49 nurses, $56 \%$ of the target population. Attrition occurred due to lack of response to requests to complete the follow-up questionnaires.

All 49 participants were qualified adult nurses, 48 were female and one was male. In all, 30 of the 49 Nurse participants had undertaken previous sexual health education. The mean number of years since nurse qualification was 17 years (SD 8.5 ) and the mean length of time working in sexual health was six years (SD 6.6).

The questionnaires were given to nurses at the beginning and the end of their engagement with the workforce development programme. Data collected included biographical data and seven free text questions inviting nurses to write in depth about their ability to make changes in practice, the perceived impact of their learning on services users and challenges faced throughout their development. Open questions were deliberately included as a strategy to identify further issues for inclusion in the interviews and focus groups and to complement responses to closed questions (O'Cathain and Thomas, 2004).

In addition, nurses were invited to attend one of a number of focus groups, held at the university, or take part in a telephone interview to explore some of the themes that were identified from the questionnaire data in more depth.

\section{Interviews}

Qualitative interviews were chosen as a method of establishing common themes between the respondents and a 'joint construction of meaning' (Gubrium and Holstein, 2002: 17). Nurse interviews were conducted within one month of the nurses completing their academic modules, enabling them to offer an additional perspective about the impact their learning had on service delivery. The interview guide was influenced by the comments made in the free text sections of the questionnaires and included questions about the impact nurse learning had on service delivery. Seven nurses undertook individual telephone interviews. These nurses worked in different settings across the South West region, including
GP surgeries, integrated sexual health clinics, contraception clinics and a minor injuries unit.

All known service managers for the organisations offering sexual health services and supporting nurses on these modules in the region were contacted by email with details of the study and invited to participate in semi-structured telephone interviews. This purposive sampling resulted in responses from six service managers. Three of their organisations offered a fully integrated sexual health service, two were partially integrated and one was entirely separate. Service manager interviews, conducted in July 2015, focussed on the preparation of nurses for practice and the impact the nurses had on service delivery or patient care. The interviews were audio recorded and transcribed and lasted 30-40 min.

\section{Focus groups}

Focus groups were chosen due to the added value of group dynamics (Kamberelis and Dimitriadas, 2005). In total, 22 nurses participated in three focus groups. Researchers facilitated the focus groups, which lasted $1 \mathrm{~h}$, were audio recorded and transcribed. Discussion was influenced by the study objectives and the findings from analysis of the qualitative questionnaire data, specifically whether the nurses felt prepared to work within integrated sexual health in practice and if they were able to influence or make changes in service delivery.

As part of each focus group the participants engaged in a diamond ranking activity to promote discussion (Clark, 2012). Diamond ranking builds on the principles of nominal group technique by attempting to gain consensus, facilitating all participants to contribute and prioritise ideas democratically (Tague, 2004). The activity focussed on the specific question, 'What impact has your learning had on service users?'. Five nurses participated in the first diamond ranking activity, nine in the second and eight in the third. The researchers had previously identified nine broad impacts that learning may have on service users from the questionnaire answers (Table 1) and participants were also able to offer their own ideas on two 'wild cards'. The nurses were asked to rank the nine impacts, in order of importance using topic cards and a diamond pattern. The top of the 
Table 1 Impacts that learning may have on service users

More comprehensive assessment

More comprehensive treatment

A one stop shop

Service users receive evidence-based clinical care

Improved signposting and communication between agencies

Better relationships so more opportunities for disclosure

Care is individualised to the needs of the patient

More flexible and accessible services

Increased quality of care

diamond represents the most important priority identified by the nurses.

\section{Data analysis}

Braun and Clarke's (2006) six-stage technique was used during the analysis of the questionnaire, interview and focus group data, beginning with familiarisation with the data (stage 1), generating initial codes (stage 2), searching for, reviewing and naming themes (stages 3,4 and 5), and writing a report (stage 6). Thematic analysis is a method in its own right due to its flexibility and in particular, the compatibility of the process with a range of different paradigms. The analysis of the data was predominantly deductive and conducted at the semantic level. This approach allowed movement from raw data to abstraction in the analytic process, focussed on the voices of participants. Four key themes were identified from the perspectives of nurses and their managers and included: new knowledge and changing practice; leadership and delivery of integrated services and impact on patient care. Opportunities were taken during the interviews and focus groups to explore in depth any opinion or experience that emerged as distinct from general consensus. Figure 1 illustrates the process of analysis.

\section{Trustworthiness}

The triangulation of information from the focus groups, interviews and questionnaires demonstrated the credibility and dependability of the data. The qualitative data were independently analysed by two of the research team (J.S., J.B.)

Primary Health Care Research \& Development 2017; 18: 270-281 and themes compared and discussed to confirm validity. The transcripts had been anonymised so the researchers were blind to the identity of the participants. Both researchers were independent to the sexual health module delivery, which offered a level of objectivity to the process and encouraged open responses from the participants. Reflexivity was incorporated at each stage of the research process in order to recognise and bring to the forefront any preconceptions, attitudes, values and beliefs held by the researchers that may influence the research process (Etherington, 2007). The potential bias inherent in a volunteer sample in the context of an assessed educational programme was mitigated by the fact that the researchers were not connected to the module delivery. The students were reassured about the confidentiality of their responses, inviting openness and honesty.

\section{Results}

\section{Nurse perspectives}

\section{New knowledge and changing practice}

The majority of respondents felt that increased knowledge and confidence legitimised their ability to question practice and suggest changes to wellestablished customs. They appreciated the opportunity to step back and look objectively at service delivery, identify areas for improvement and also barriers to change. Comments indicated that participation in the learning had given them a greater awareness of the evidence base and the wider influences on sexual health.

I have a better understanding of what is meant by an integrated service and how providing such a service improves overall experience and outcome for the client. I have been able to demonstrate areas for improvement within my current chlamydia screening role but equally barriers that impact on implementing change.

(Nurse 016)

The module has expanded my knowledge and understanding of both individual and organisational factors which can impact on sexual and reproductive health, I feel it has 


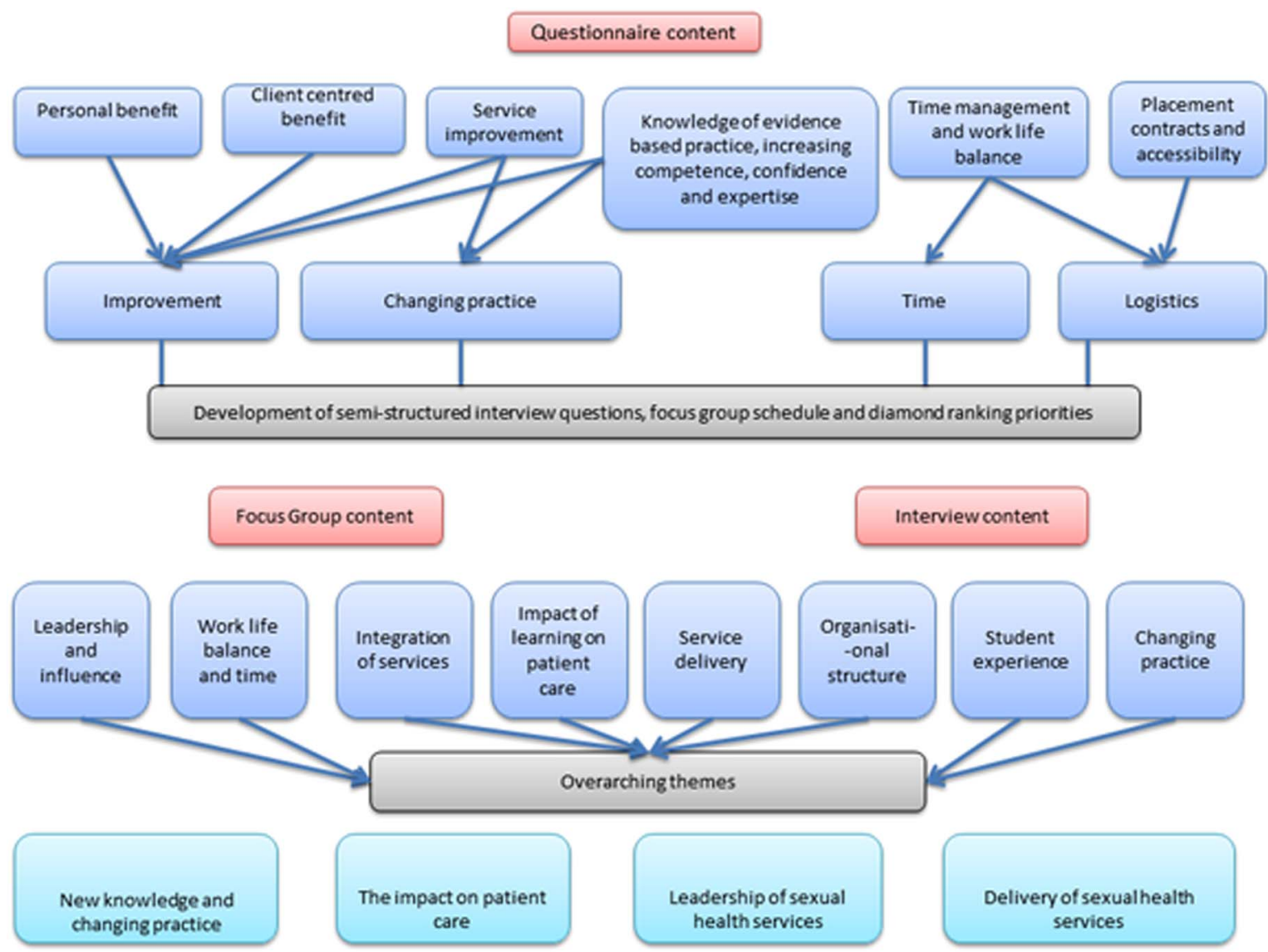

Figure 1 Flowchart of data analysis

made me a more rounded professional that is better able to meet patient needs.

(Nurse 083)

Several nurses identified that by changing practice they were now able to provide a 'better service'. This was attributed to their personal skills, such as being able to facilitate more open conversations with clients to encourage disclosure, and their increased knowledge, which allowed them to offer a more holistic and integrated service to clients. This included more in depth and thorough consultations, improved diagnosis and earlier treatment intervention; client experience was enhanced because the practitioner could offer a broader and higher quality of care. In addition, nurses felt that their ability to offer a 'one stop shop' would in turn reduce waiting times, increase access to services for patients and provide a more seamless service but only if the context of service delivery could facilitate integrated care.

It will provide a holistic and hopefully seamless service in the future, once the service itself is equipped to offer integrated sexual healthcare.

(Nurse 003)

I will be able to advise and deal with a wider range of sexual health issues in one consultation and know that I am providing evidence-based practice for service users. Better quality and patient experience.

(Nurse 032) 
The context of service delivery greatly influenced the nurses' perceptions of the quality of the service they were offering and is discussed below.

\section{Integrated service delivery and leadership}

Some nurses worked in fully integrated services, some co-located and others entirely separate. There was a unanimous belief expressed that integration was a positive step, reinforced by the new learning, but also recognition of the challenges to achieving this, both at individual and organisational level. Structural factors such as the way services were commissioned, fragmentation of services as a result of failed service tenders or the introduction of new providers, and managing clinics in isolated geographical areas all presented further challenges to maintaining the momentum of change.

Nurses who were working in non-integrated services expressed frustration at knowing about best practice but feeling they had no influence over the integration agenda. They felt that services were hierarchical in that decisions were taken by leaders or managers rather than through democratic consultation with staff or service users. The impact of financial considerations and commissioning structures was perceived to override the best interests of clinical staff and patients and thwart integration. Central to supporting change was effective leadership and leaders who were passionate and committed to the introduction of integrated services and education of staff to deliver those services. Although the sexual health learning equipped participants to understand the political landscape, they did not feel it equipped them to influence change and they did not currently see themselves as clinical leaders in their field of practice.

It frustrates the hell out of me now because I know what we should be able to do and it makes me quite a frustrated practitioner because I can't do ... there is too much politics and there's too many issues for me to be able to do what I want to do so it does make it quite a fragmented time, quite frustrating.

(Focus Group 2, participant 14)

You get the feeling that there is a lot going on behind the scenes that we are not told about and it would just be nice if we were kept in the loop about it we're not even asked questions about how we feel because most of us have got really good ideas about how an integrated service should and could be run but nobody really asks us our opinion.

(Focus Group 2, participant 11)

However, there was a sense that nurses were so committed to the integrated agenda that they would find their own ways of subverting the system so that they could offer a holistic service; patients received both sexual health and contraception advice treatment despite the challenges.

I guess people are developing their own ways of integrating and delivering the whole lot in one go.

(Nurse 001)

I probably practice an integration model whereby I provide the services but not necessarily all at the same time ... so they get an integrated service [from me] but it's not necessarily in the same consultation at the same time.

(Nurse 008)

This exploitation of professional autonomy links with the concept of street-level bureaucracy (Lipsky, 1980), which is explored further in the discussion.

\section{Impact on patient care}

The results of the diamond ranking activity can be seen in Figure 2. Group 3 did not reach a consensus on their lowest priority so left this area blank.

All three groups agreed that the programme had enabled them to provide more comprehensive assessment for their service users, an activity which is influenced to a large extent by the skills of the individual practitioner. Although they would like to have made an impact on the service user experience to a greater extent, structural restraints, such as not offering an integrated service, limited their impact, hence the positioning of the 'one stop shop' card towards the bottom of the diamond.

\section{Clinical service manager perspectives}

\section{Leadership of integrated service delivery}

All managers accepted that integrated service delivery was the most favourable model, primarily 


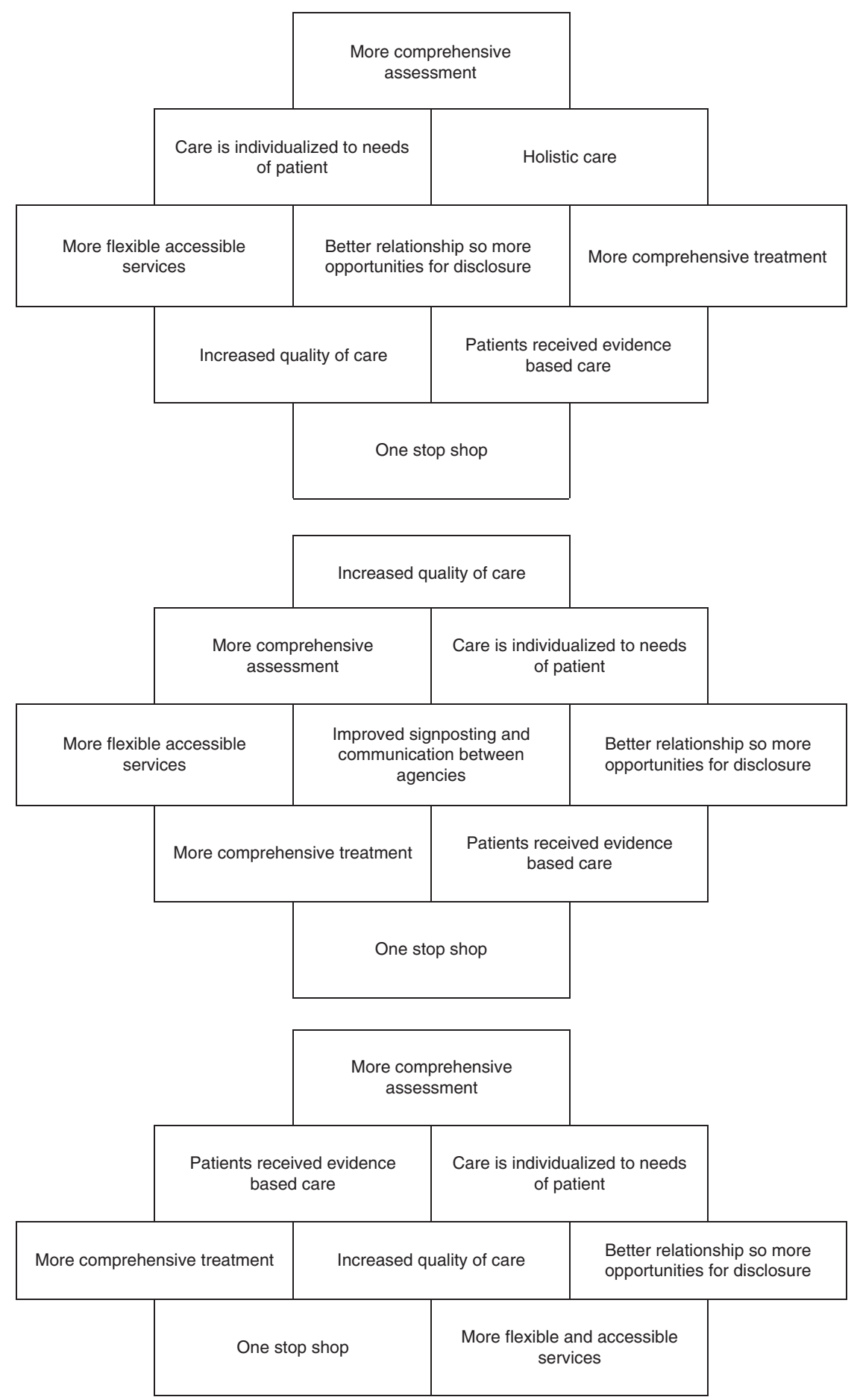

Figure 2 Diagrams to illustrate the results of the three diamond ranking exercises

Primary Health Care Research \& Development 2017; 18: 270-281 
because of the benefit to the patients. Even in those services that were not described as integrated, mangers attempted to implement systems that emulated the model.

We saw the writing on the wall about seven years ago and we realised if you are in that area of the anatomy and they had unprotected sex the probability is that they need some contraception so it seemed crazy to make them go to a different service to get some contraception so anybody that needs contraception opportunistically we will give it to them.

(Manager 3)

A sub-theme in this section was that integration of sexual health and contraception services was described as a journey, which required a certain amount of tenacity from the managers. Some areas had successfully completed the transition and others had tried and failed, only to try again with a different approach.

I think mainly for the patients you know it's always patient focused what we want to do and it definitely would be the best option for patients but yes for various reasons unfortunately we've got close a few times but it's never actually happened.

(Manager 1)

I am a big, big strong believer in that and think that's something that we pushed you know with the [Trust name] for quite a few years now and it was a massive turnaround for a lot of staff to that way of working who were historically weren't working in that way.

(Manager 2)

Mangers identified that the experience nurses had of integrated services could affect their perspective. If a nurse came from either a contraceptive or GUM clinic, a practice placement in a fully integrated service had the potential to be unsettling. However, this experience could also be beneficial in terms of motivation and innovation.

They are doing the course and then they are going back into a service that isn't integrated and it's a real frustration because once you start to work in an integrated way it's very difficult to see how you cannot continue to do that.

(Manager 4)

I have got a couple of staff ... one has worked in an integrated service before ... and had a much more strategic view and a way of thinking and my nurse that's doing this course, they are starting to actually see and come up with suggestions and they are starting innovative practice.

(Manager 5)

The perspective of the nurses and their established colleagues in the sexual health workforce was key to influencing and implementing change. The impact this had on service delivery is discussed below.

\section{Delivery of services}

The managers highlighted that the increased expertise of the nurses led to innovation and change in the workplace. This was not met with unanimous acceptance by existing staff members and this resistance was an additional challenge for managers. Managers valued the opportunity that the workforce development programme allowed nurses to reflect on how they were working and consider the issues from a wider perspective. They did not suggest that the skills the nurses were developing were leadership skills but acknowledged that they were more confident to suggest service developments and to have a greater ability to think strategically about the issues that affect both individuals and services on completion of the workforce development programme. One participant emphasised the fact that it was not the clinical competence that enabled them to develop professionally but the "peripheral learning' that encouraged the nurses to become more professionally motivated.

I am not sure leadership is quite the right term perhaps maybe it just gives them you know maybe slightly more confidence to actually come and discuss things ... I think it just sort of opens your mind up wider doesn't it ... it sort of gives somebody time to sit down and really reflect on how they're working.

(Manager 1) 
It makes people more open minded and they actually see their service in the context of people's lives as well and what they are struggling with and why they can't get there on time and why some people are booked and some people are drop in, it's that whole ... it's that bigger picture of patients as well as service.

(Manager 5)

\section{Discussion}

Allotey et al. (2011) argue that working in sexual and reproductive health is not merely about clinical competency but also about negotiating the complexities of a speciality steeped in social values, ideology, religion and morality. The fact that sexual ill health is also inextricably linked with inequality, disadvantage and debate about human rights makes it inherently political. The policy agenda (DH, 2013a) in the UK reiterates the need for broader-based service delivery, which in turn requires a broader-based education programme for practitioners in the field of sexual health, to enable holistic work with patients (Kane and Wellings, 2003). The clinical service managers in this study recognised the value of 'peripheral learning', described as learning outside of clinical competency and core skills but still essential to developing motivated and rounded professionals, who could consider the service not only on the individual but also the strategic level. This reinforces the desire to move away from a medically dominated discourse on sexual health to one that acknowledges the importance of a holistic, public health perspective. The nurses discussed the role of new learning in enabling them to negotiate the political landscape but expressed frustration at their lack of influence and ability in the integration agenda, exposing a clear dichotomy between the intentions of policy and the reality of practice.

Both service managers and nurses believed that integrated service delivery was the appropriate model but both sets of participants expressed frustration at the slow pace of change. Once the nurses had experienced working in an integrated way they found it hard to reconcile separate service provision. However, there was a sense that nurses were so committed to the integrated agenda that they found their own ways of subverting the system so that they could offer a holistic service; patients received both sexual health and contraception advice and treatment despite the challenges. This suggests at least some change in workforce culture in the last five years and contradicts the nurses' perception that they had no agency in how services were delivered.

The actions of the nurses in this study can be related to the concept of street-level bureaucracy (Lipsky, 1980), where employees of government agencies (in this case the NHS) exercise substantial discretion in the way they carry out their roles, in part to overcome conflicts between their own beliefs and commitments, and the demands of their jobs and in part to fulfil an obligation (perceived or otherwise) to remain advocates for their patients. This is only possible due to the high level of professional judgement that specialist practitioners such as sexual health nurses are expected to exercise and the 'human' dimension of the work that they do. The nurses must respond with compassion and flexibility to the needs of the individual patient (Cummings, 2012) and such idiosyncratic response may unintentionally undermine the broader organisational goals. Summers and Semrud-Clikeman (2000) found that school psychologists, when faced with conflicts between their professional integrity, beliefs and organisational context, developed coping strategies that included circumventing the job demands to provide necessary services to children and their parents. Ironically, the actions of the sexual health nurses in this study may in turn influence local policy, demonstrating the very leadership skills that participants reported failing to achieve.

The outcome of the diamond ranking activity was a further indication that the messages from national policy are not necessarily being enacted at local level. The nurses gave priority to impacts on services users that they felt were within their locus of control (Rotter, 1966). Those impacts that required wider organisational or structural support, were not identified in the diamond, or were given low priority. It may be that their focus on individual, patient-centred care was a way of reconciling themselves with the structural barriers that they experienced.

In reality, policy making and implementation is a complex activity that is influenced by a range of factors (Bunn and Kendall, 2011). Evans et al. (2013) discussed the influences on commissioners 
and service managers when implementing policy at local level and highlighted the important part that financial implications, government targets and local political issues played in the decision-making processes, often influencing to a greater extent than research evidence, resulting in policy that is sometimes ideological and contradictory. The sexual health agenda articulates holistic, integrated and accessible care but health budgets and service organisation structures undermine this ambition by presenting challenges to integrated delivery. Rütten et al. (2009) also propose that organisations can exhibit different levels of 'readiness' to adopt policy, which incorporate factors such as supportive environments, organisational goals, organisational resources, organisational duties and external 'windows of opportunity' that may be present. These complex structural and demographic challenges are not limited to the United Kingdom, as illustrated by the challenges of increasing utilisation of integrated sexual health services internationally. Smit et al.'s (2012) research in South Africa highlighted that issues such as under-funding, territorialism and weak referral systems impact on implementation at service level, despite integrated sexual health services being a policy priority. Clearly, practitioners face a number of barriers to introducing new evidence and policy in to practice, not least authority and support for innovation (Gerrish et al., 2007).

\section{Conclusion}

This study emphasises the need for appropriate sexual health education to support the transition to delivering integrated services to patients. However, clinical education alone is insufficient to fulfil the sexual health agenda. Future planning should also include leadership and change management theory and practice. Nurses demonstrated the use of intuitive leadership characteristics but lacked the confidence to acknowledge their ability to influence the integration agenda. More explicit leadership development may enable practitioners to initiate and manage service development and influence decision makers in their organisation.

Commitment to integrated service delivery should continue. A supportive culture is imperative to enable practitioners to propose and implement change in practice. This cultural shift is not only important at the level of service providers; policy makers should recognise the challenges presented by current fragmented commissioning structures to delivering integrated sexual health services and the impact this has on practitioners and service users. Sexual health as a specialism is in a state of flux and practitioners need to be equipped not only to work holistically with patients and clients but also to develop a resilience that allows them to play a central part in developing excellent, accessible services that meet the needs of the users.

\section{Limitations}

The study would have been enriched by the involvement of service users, particularly to gain their perspective on improvements to service delivery by nurses who had completed the learning. In addition, the perspectives of commissioners would have broadened the scope of the study.

\section{Acknowledgements}

The authors would like to acknowledge the contribution of all the participants in this study, the valuable advice of the members of the advisory committee and the financial support of Health Education South West.

\section{Conflicts of Interest}

The authors declare that they have no potential conflicts of interest with respect to the research, authorship and/or publication of this article.

\section{References}

Allotey, P., Diniz, S., DeJong, J., Delvaux, T., Gruskin, S. and Fonnf, S. 2011: Sexual and reproductive health and rights in public health education. Reproductive Health Matters 19, $56-68$.

Anderson, J. 2014: Integrated Sexual Health Services. The Health Service Journal 26, 6414.

Braun, V. and Clarke, V. 2006: Using thematic analysis in psychology. Qualitative Research in Psychology 3, 77-101.

British Education Research Association 2011. Ethical guidelines for educational research. London: BERA.

Bunn, F. and Kendall, S. 2011: Does nursing research impact on policy? A case study of health visiting research and UK health policy. Journal of Research in Nursing 16, 169-91. 
Clark, J. 2012: Using diamond ranking as visual cues to engage young people in the research process. Qualitative Research Journal 12, 222-37.

Cummings, J. 2012: Compassion in practice: nursing, midwifery and healthcare staff, our vision and strategy. Retrieved 7 December 2015 from https://www.england.nhs.uk/nursingvision/compassion/.

Department of Health (DH) 2013a: A framework for sexual health improvement in England. London: Crown copyright.

Department of Health (DH) 2013b: Integrated sexual health services: NHS specification. A suggested service specification for integrated sexual health services. London: Crown copyright.

Dickinson, C., Attawell, K. and Druce, N. 2009: Progress on scaling up integrated services for sexual and reproductive health and HIV. Bull World Health Organ 87, 846-51.

Etherington, K. 2007: Ethical Research in Reflexive Relationships. Qualitative Inquiry 13, 599-616.

Evans, B., Snooks, H., Howson, H. and Davies, M. 2013: How hard can it be to include research evidence and evaluation in local health policy implementation? Results from a mixed methods study. Implementation Science 8, 17.

Gerrish, K., Ashworth, P., Lacey, A., Bailey, J., Cooke, J., Kendall, S. and McNeilly, E. 2007: Factors influencing the development of evidence-based practice: a research tool. Journal of Advanced Nursing 57, 328-38.

Gubrium, J.E. and Holstein, J.A. 2002: From the individual interview to the interview society. In Gubrium J.E. and Holstein J.A., editors Handbook of interview research: context and method. Thousand Oaks, CA: Sage, 3-32.

Ivankovich, M.B., Fenton, K.A. and Douglas, J.M. 2013: Considerations for National Public Health Leadership in Advancing Sexual Health. Public Health Reports 128 (Suppl), 102-10.

Kamberelis, G. and Dimitriadas, G. 2005: Focus groups: strategic articulations of pedagogy, politics, and inquiry. In Denzin, N.K. and Lincoln, Y.S., editors The Sage handbook of qualitative research, third edition. Thousand Oaks, CA: Sage, 887-907.

Kane, R. and Wellings, K. 2003: Staff training in integrated sexual health services. Sexually Transmitted Infections Journal 79, 354-56.

Lipsky, M. 1980: Street-level bureaucracy. New York: Sage.
McNall, A. and Kain, K. 2010: Sexual Health Advising - Developing the Workforce. Increasing capacity in NHS North East Region through workforce development innovation funding. Final Report, April, NHS North East, York.

O'Cathain, A. and Thomas, K. 2004: 'Any other comments?' Open questions on questionnaires - a bane or a bonus to research? BMC Medical Research Methodology $4,25$.

Public Health England 2014: South West Public Health Observatory sexual health balanced scorecard. Retrieved 13 May 2016 from http://www.apho.org.uk/addons/_118371/ atlas.html.

Public Health England (PHE) 2015: Making it work: a guide to whole system commissioning for sexual health, reproductive health and HIV. Retrieved 6 May 2016 from https://www. gov.uk/government/publications/commissioning-sexual-healthreproductive-health-and-hiv-services.

Rotter, J.B. 1966: Generalized expectancies of internal versus external control of reinforcements. Psychological Monographs $80,609$.

Rutaremwa, G. and Kabagenyi, A. 2016: Utilization of integrated HIV and sexual and reproductive health services among women in Uganda. BMC Health Services Research $16,494$.

Rütten, A., Röger, U., Abu-Omar, K. and Frahsa, A. 2009: Assessment of organizational readiness for health promotion policy implementation: test of a theoretical model. Health Promotion International 24, 243-51.

Smit, J.A., Church, K., Milford, C., Harrison, A.D. and Beksinska, M.E. 2012: Key informant perspectives on policy and service-level challenges and opportunities for delivering integrated sexual and reproductive health and HIV care in South Africa. BMC Health Services Research 12, 48.

Summers, A. and Semrud-Clikeman, M. 2000: Implementation of the IDEA by School Psychologists: an exploratory study using the theory of street-level bureaucracy. School Psychology Quarterly 15, 255-78.

Tague, N. 2004: The Quality Toolbox, Second Edition. Milwaukee: ASQ Quality Press, pp. 364-365.

Temmerman, M., Khosler, R. and Say, L. 2014: Sexual and reproductive health and rights: a global development, health, and human rights priority. The Lancet 384, e30-31. 